\title{
Quantitative Trait Loci for Slow-Rusting Resistance to Leaf Rust in Doubled-Haploid Wheat Population CI13227 × Lakin
}

\author{
Yue Lu, Robert L. Bowden, Guorong Zhang, Xiangyang Xu, Allan K. Fritz, and Guihua Bai ${ }^{\dagger}$
}

First and fifth authors: Department of Agronomy, Kansas State University, 2002 Throckmorton Hall, Manhattan 66506; second and sixth authors: Hard Winter Wheat Genetics Research Unit, United States Department of Agriculture-Agricultural Research Service (USDA-ARS), 4008 Throckmorton Hall, Manhattan, KS 66506; third author: Agricultural Research Center-Hays, Kansas State University, Hays 67601; and fourth author: Wheat, Peanut and Other Field Crop Research Unit, USDA-ARS, Stillwater, OK.

Accepted for publication 5 June 2017.

\begin{abstract}
CI13227 is a U.S. winter wheat line with adult-plant slow-rusting resistance that has been the subject of several studies on the characteristics and components of slow rusting. Previous genetic studies used different populations and approaches and came to different conclusions about the genetic basis of resistance in CI13227. To clarify the situation, a new doubledhaploid (DH) population of CI13227 $\times$ Lakin was produced and a linkage map was constructed using 5,570 single-nucleotide polymorphism (SNP) markers derived from wheat $90 \mathrm{~K}$ SNP assays and 84 simple sequence repeat markers. Three quantitative trait loci (QTL) were identified for three slow-

2DS, designated QLr.hwwg-2DS, explained 11.2 to $25.6 \%$ of the phenotypic variation. It was found in the same position as a slow-rusting QTL in the CI13227 $\times$ Suwon 92 population in a previous study and, thus, verified the 2DS QTL. The QTL on chromosome 7BL explained 8.1 and $19.3 \%$ of the phenotypic variation and is likely to be Lr68. The other two QTL showed a minor effect on some of the traits evaluated in a single experiment. Flanking SNP closely linked to all QTL were converted to Kompetitive allele-specific polymerase chain reaction markers that can be used in marker-assisted selection to transfer these QTL into adapted wheat cultivars.
\end{abstract} rusting traits on chromosome arms 2DS, 7AL, and 7BL from CI13227. A fourth QTL mapped on chromosome 3BS was from Lakin. The QTL on
Additional words: SNP marker, Triticum aestivum.
Leaf rust, caused by Puccinia triticina Erikss., is an important disease of wheat (Triticum aestivum L.) globally. Leaf rust occurs more frequently and in larger areas worldwide than wheat stem rust (P. graminis f. sp. tritici) or stripe rust ( $P$. striiformis f. sp. tritici) (Bolton et al. 2008). Yield losses caused by leaf rust result mainly from premature leaf senescence and poorly developed kernels. Genetic resistance is the preferred method to reduce losses from rust epidemics. Although more than 70 leaf rust resistance genes have been identified in wheat (McIntosh et al. 2013), most of them confer race-specific resistance in a qualitative, gene-for-gene manner. They often lose effectiveness within a few years of deployment in a large area that imposes selection for virulent races of the rust pathogen. Adult-plant slow-rusting resistance, which is a type of quantitative resistance, has proven to be more durable than qualitative resistance (Singh et al. 2000, 2011).

Cultivars with adult-plant slow-rusting resistance typically display a compatible infection type (IT) at the seedling stage; however, plants become more resistant as the plant reaches the reproductive phase. A key feature is that the rate of disease progress is much slower than in susceptible cultivars (Shaner and Finney 1980). A slow-rusting cultivar is usually characterized as having a longer latent period (LP), lower receptivity, smaller pustule size, and less spore production than a susceptible cultivar. Together, these slow-rusting traits retard rust disease progress and result in a smaller area under the disease progress curve (AUDPC) and lower final disease severity (FS) in resistant cultivars than in susceptible

†Corresponding author: G. Bai; E-mail: guihua.bai@ars.usda.gov

This is contribution number 17-002-J from the Kansas Agricultural Experiment Station.

This article is in the public domain and not copyrightable. It may be freely reprinted with customary crediting of the source. The American Phytopathological Society, 2017. cultivars (Herrera-Foessel et al. 2012). The genes conditioning slow rusting usually show additive effects, and pyramiding multiple genes may result in high levels of resistance up to near immunity (Singh et al. 2000, 2011).

Much progress has been made in searching for slow-rusting resistance to leaf rust in wheat. To date, four slow-rusting adult plant resistance (APR) genes for leaf rust have been named: $L r 34, \operatorname{Lr} 46$, Lr67, and Lr68. Lr34 was first reported on chromosome 7D in Brazilian 'Frontana' wheat (Dyck et al. 1966) and discovered to confer additional resistance to stripe rust $(\mathrm{Yr} 18)$, stem rust $(\mathrm{Sr} 57)$, powdery mildew (Pm38), and leaf tip necrosis ( Ltn 1 ) (Lillemo et al. 2008; Krattinger et al. 2009). Singh et al. (1998) identified $L r 46$ on chromosome 1B from 'Pavon 76'. It is colocated with slow-rusting gene $\mathrm{Yr} 29$ for stripe rust, $S r 58$ for stem rust resistance, and $P m 39$ for powdery mildew resistance (McIntosh et al. 2013; William et al. 2003). Herrera-Foessel et al. $(2011,2014)$ mapped the third slowrusting resistance gene, $\operatorname{Lr} 67$, to the interval between simple sequence repeat (SSR) markers X $g w m 165$ and Xgwm 192 on chromosome 4DL, which is also associated with resistance to stem rust (Sr55), stripe rust (Yr46) and powdery mildew (Pm46). Herrera-Foessel et al. (2012) found a new APR quantitative trait loci (QTL) for leaf rust resistance in 'Parula' wheat on chromosome 7BL and designated it Lr68. Among these slow-rusting APR genes, Lr34 (Krattinger et al. 2009) and Lr67 (Moore et al. 2015) have been cloned. Because slow-rusting APR genes have been durable as well as additive and pleiotropic in their effects, additional sources of slow-rusting resistance are of great interest to breeders.

The winter wheat line CI13227 from Purdue University was originally developed by R. M. Caldwell and was reported to have exceptionally good slow-rusting resistance (Lee and Shaner 1985; Shaner and Finney 1980). Slow-rusting resistance in CI13227 was believed to be derived from Wabash, a landrace from Indiana, that was collected in the 1940s (Clark 1940). The distribution of LP among the recombinant inbred lines (RIL) of CI13227/Suwon 92 suggested that one major gene and three minor genes probably 
condition LP (Shaner et al. 1997). Xu et al. (2005c) used the same population and phenotypic data, and identified a major QTL for LP, QLrlp.osu-2DS, that accounted for 35 to $48 \%$ of the phenotypic variation. QLrlp.osu-2B and QLrlp.osu-7BL each explained 11 to $16 \%$ of the variation in LP. In field studies with the same population, Xu et al. (2005b) identified QLr.osu- $2 B$ and QLr.osu-7BL that were associated with lower AUDPC, FS, and infection rate (daily disease progress rate) and each explained 13 to $21 \%$ of the variation. QLrid. $o s u-2 D S$ was associated with longer infection duration (length of rust sporulation period) and explained 21 to $26 \%$ of the variation (Xu et al. 2005b). However, Kolmer et al. (2012) did not find any of these QTL in the RIL population developed from Thatcher*3/ CI13227. Instead, they detected seedling resistance gene $L r 3 \mathrm{ka}$ and adult-plant slow-rusting gene $L r 46$ in the population in two field experiments.

To help resolve the inconsistent results, we reexamined the QTL in CI13227 using a new doubled-haploid (DH) mapping population and better marker coverage. Recently, high-density SNP arrays have been developed for wheat and widely utilized in QTL mapping and genome-wide association studies (Cavanagh et al. 2013; Wang et al. 2014). A high-density genetic map is essential for fine mapping of QTL and delimiting the causal genes to a narrow genetic interval ( $\mathrm{Li}$ et al. 2014). Also, high-density SNP maps provide an opportunity to identify SNP tightly linked to these QTL for pyramiding them using marker-assisted selection (MAS).

The objectives of this study were to (i) verify QTL for adult-plant slow-rusting resistance in CI13227, (ii) identify SNP markers tightly linked to the QTL, and (iii) convert chip-based SNP into breederfriendly Kompetitive allele-specific polymerase chain reaction (KASP) markers so that they can be used in MAS to improve APR in wheat.

\section{MATERIALS AND METHODS}

Plant materials. A DH population with 181 lines was developed by Heartland Plant Innovations (Manhattan, KS) from $\mathrm{F}_{1}$ seed from a cross between Lakin (Arlin/KS89H130) and CI13227 (Wabash/ American Banner//Klein Anniversario). CI13227 from Purdue University has a high level of slow leaf-rusting resistance with a long LP (Shaner and Finney 1980; Shaner et al. 1997), whereas Lakin from Kansas State University is moderately susceptible to leaf rust.

Selection of a seedling-virulent $\boldsymbol{P}$. triticina culture. In order to test for APR without interference from resistance expressed at the seedling stage, a $P$. triticina culture was sought that was virulent on seedlings of both parents of the DH population. Seed were planted in 4.5-cm-diameter pots containing Metro-Mix 360 growing mix (Hummert International, Earth City, MO) and were grown at $20 \pm 3^{\circ} \mathrm{C}$ in the greenhouse. Urediniospores stored at $-80^{\circ} \mathrm{C}$ were heat-shocked at $42^{\circ} \mathrm{C}$ for 6 min before inoculation. Ten-day-old seedlings in each pot were inoculated by spraying approximately $0.3 \mathrm{ml}$ of a urediniospore suspension at a concentration of approximately $6 \times 10^{6}$ spores/ml in Soltrol 170 isoparaffin light mineral oil (Chevron Phillips Chemical Company LLC, The Woodlands, TX). After the oil had evaporated for $10 \mathrm{~min}$, the inoculated seedlings were incubated in a dew chamber at $100 \%$ relative humidity and 20 $\pm 2^{\circ} \mathrm{C}$ for $20 \mathrm{~h}$. IT were recorded 14 days after inoculation using the 0-to-4 Stakman scale (Roelfs et al. 1992). The P. triticina cultures used for seedling inoculation included race MFPSB (virulent to $L r l$, $L r 3 a, L r 24, L r 26, L r 3 k a, L r 17, L r 30, L r B, L r 10$, and $L r 14 a$ ), race PNMRJ (virulent to $L r 1, L r 2 c, L r 3 a, L r 9, L r 24, L r 3 k a, L r 30, L r B$, Lr10, Lr28, and Lr39), and four other uncharacterized isolates that were collected from the field in 2010, 2011, 2013, and 2014.

Evaluation of leaf rust responses at the adult plant stage. Both parents and the DH lines were evaluated for LP, FS, and AUDPC in greenhouse experiments at Kansas State University, Manhattan in fall 2012 and spring 2013 using a randomized complete block design with two replications. After heading, four to five plants per replication were inoculated with approximately $0.3 \mathrm{ml}$ of a spore suspension of the selected P. triticina isolate at a concentration of approximately $6 \times 10^{6}$ spores $/ \mathrm{ml}$. The inoculated plants were kept overnight in a misting chamber at 20 to $25^{\circ} \mathrm{C}$ to maintain high moisture for infection. Infected plants were moved to greenhouse benches for rust development at $22 \pm 4^{\circ} \mathrm{C}$. LP was measured as the number of days from inoculation to the appearance of the first uredinium. Rust severity on flag leaves was evaluated by the modified Cobb scale (Peterson et al. 1948; Roelfs et al. 1992) to estimate a percentage of leaf area covered by rust pustules at 7, 10, 13, and 15 days after inoculation. An AUDPC was calculated for each plant based on the rust severities using the formula AUDPC $=$ $\Sigma(i=1, n-1)\left[\left(t_{i+1}-t_{i}\right)\left(y_{i}+y_{i+1}\right) / 2\right]$, where $t$ accounts for the day after inoculation, $y$ accounts for rust severity, and $n$ accounts for the number of days on which rust severity was collected. At 15 days after inoculation, FS was evaluated as previously described (Campbell and Madden 1990).

Molecular marker analysis. Two-week-old leaves were harvested from the parents and DH lines for DNA extraction using the cetyltrimethylammonium bromide method (Sun et al. 2009). SNP genotyping was performed using wheat $90 \mathrm{~K}$ SNP assays developed by Illumina Inc. (San Diego, CA) and assembled by the International Wheat SNP Consortium (Cavanagh et al. 2013; Wang et al. 2014). The genotyping assays were performed at the United States Department of Agriculture (USDA) Small Grains Genotyping Laboratory in Fargo, ND, and SNP genotypes were determined using GenomeStudio (v2011.1; Illumina, San Diego, CA).

A selected set of 384 SSR primers was screened for polymorphism between the parents, and polymorphic SSR markers were used to screen the DH population. For each SSR reaction, a 20- $\mu 1$ polymerase chain reaction (PCR) mix containing $50 \mathrm{ng}$ of genomic DNA, $0.2 \mathrm{mM}$ each dNTP, $1 \times$ ammonium sulfide PCR buffer, $0.1 \mu \mathrm{M}$ forward primer, $0.15 \mu \mathrm{M}$ reverse primer, $2.5 \mathrm{mM} \mathrm{Mg}^{2+}$, $0.05 \mu \mathrm{M}$ dye-labeled M13 primer, and $1 \mathrm{U}$ of Taq polymerase. A touchdown program for PCR amplification started at $95^{\circ} \mathrm{C}$ for $5 \mathrm{~min}$, followed by five cycles of $45 \mathrm{~s}$ at $95^{\circ} \mathrm{C}, 5 \mathrm{~min}$ of annealing at $68^{\circ} \mathrm{C}$ that decreased by $2^{\circ} \mathrm{C}$ in each subsequent cycle, and $1 \mathrm{~min}$ of extension at $72^{\circ} \mathrm{C}$. In the subsequent five cycles, the annealing time was reduced to $2 \mathrm{~min}$, with a decrease of $2^{\circ} \mathrm{C}$ in each subsequent cycle. PCR was continued for an additional 25 cycles of $45 \mathrm{~s}$ at $94^{\circ} \mathrm{C}, 2 \mathrm{~min}$ at $50^{\circ} \mathrm{C}$, and $1 \mathrm{~min}$ at $72^{\circ} \mathrm{C}$, with a final extension at $72^{\circ} \mathrm{C}$ for $5 \mathrm{~min}$. PCR products were detected using an ABI Prism 3730 DNA Analyzer (Thermo Fisher Scientific Inc., Waltham, MA) and fragment sizes were scored using GeneMarker (version 1.97; SoftGenetics, LLC, State College, PA).

Linkage map construction and QTL identification. A genetic map was constructed using the Kosambi function (Kosambi 1943) with a minimal logarithm of the odds (LOD) threshold of 3.0 and the regression mapping algorithm in JoinMap 4.1 (Van Ooijen 2011). Markers significantly distorted from the expected segregation ratio $(P<0.0001)$ were excluded from the analysis. Composite interval mapping (CIM) in IciMapping 3.3 (http://www.isbreeding. net) was used to identify significant QTL at a walking speed of 1.0 centimorgan (cM). An LOD score of 2.75 was the threshold value for claiming a significant QTL across all traits $(P=0.05)$ based on the results of 1,000 permutations.

Statistical analysis. Analyses of variance (ANOVA) were performed for AUDPC, FS, and LP to determine significance of the difference between the parents using the software Statistical Product and Service Solution 15.0 for Windows (SPSS Inc., Chicago). Data from the 2 years of tests on the DH population were analyzed for differences in the slow-rusting traits among genotypes using a mixed-model procedure (PROC MIXED) of SAS 9.4 software.

Conversion of SNP to KASP markers. SNP that were closely linked to QTL were converted to KASP assays. New KASP primers were designed using Primer 3.0 (http://bioinfo.ut.ee/primer3-0.4.0/) and evaluated for polymorphisms between the two parents before genotyping the mapping population. KASP assays were performed in a $6-\mu$ l reaction volume ( $3 \mu \mathrm{l}$ of $2 \times$ KASP Master Mix, $0.0825 \mu \mathrm{l}$ of 
KASP primer mix, and $3 \mu \mathrm{l}$ of genomic DNA at $25 \mathrm{ng} / \mu \mathrm{l})$ and data were analyzed in an ABI 7900HT Real-Time PCR System (Thermo Fisher Scientific Inc.) following the instructions for KASP analysis (http://www.lgcgroup.com).

\section{RESULTS}

Seedling reactions of the parents to $\boldsymbol{P}$. triticina isolates. CI13227 showed resistant reactions $(<2+)$ to all the isolates except MFPSB, which was virulent on both the parents. The MFPSB culture was used for all subsequent APR tests.

Slow leaf rusting resistance in the parents and $\mathrm{DH}$ population. CI13227 had significantly lower FS and AUDPC and longer LP than the susceptible parent Lakin $(P<0.01)$ (Table 1$)$. The DH lines showed continuous distributions for all three traits measured (Fig. 1 ), indicating the quantitative nature of leaf rust resistance. Transgressive segregation was observed for all traits in both experiments (Fig. 1), suggesting that both of the parents might contribute resistance QTL.

Correlation coefficients for AUDPC $(r=0.61), \mathrm{FS}(r=0.61)$, and LP $(r=0.41)$ were highly significant $(P<0.01)$ between the two experiments. Among these traits, the correlation coefficient between

TABLE 1. Area under disease progress curve (AUDPC), final disease severity (FS), and latent period (LP) of CI13227, Lakin, and their doubled-haploid (DH) population $(n=181)$ in 2012 fall $(2012 F)$ and 2013 spring (2013S) greenhouse experiments

\begin{tabular}{lcclc}
\hline Population & Experiment & AUDPC & FS $(\%)$ & LP (days) \\
\hline CI13227 $^{\mathrm{a}}$ & $2012 \mathrm{~F}$ & 96.7 & 28.8 & 11 \\
& $2013 \mathrm{~S}$ & 92.3 & 27 & 11 \\
Lakin & $2012 \mathrm{~F}$ & 277.4 & 65.3 & 7 \\
& $2013 \mathrm{~S}$ & 266.8 & 80.8 & 7 \\
DH means & $2012 \mathrm{~F}$ & 178.7 & 46.6 & 8 \\
& $2013 \mathrm{~S}$ & 205 & 47.7 & 9 \\
DH ranges & $2012 \mathrm{~F}$ & $5.67-557.1$ & $5.0-98.0$ & $7-13$ \\
& $2013 \mathrm{~S}$ & $4.4-531.1$ & $4.9-94.2$ & $7-15$
\end{tabular}

a All three trait values for CI13227 were significantly different from those of Lakin at $P<0.01$ in each experiment.
AUDPC and FS $(r=0.96, P<0.0001)$ was the highest. LP was negatively correlated $(P<0.0001)$ with AUDPC and FS, with correlation coefficients of -0.91 and -0.89 , respectively.

Linkage map construction. Together with 84 SSR markers, 5,570 of 6,415 polymorphic SNP analyzed in the DH population were placed in a linkage map. The map consisted of 44 linkage groups with at least five markers per group, and represented all 21 chromosomes at a total length of $4,670.1 \mathrm{cM}$, with an average interval of $0.84 \mathrm{cM}$ between markers. The B genome had the most mapped markers (46.3\%), followed by the A genome (43.7\%) and D genome (10\%). Chromosome 4B had the most markers (186) at a density of 1.15 markers/cM, and chromosome $6 \mathrm{D}$ had the least at a density of 0.15 markers/cM.

QTL analysis. CIM detected four QTL on chromosomes 2D, 7B, 7A, and 3B for at least two of the three traits measured (Table 2). One QTL on chromosome 2D, designated as QLr.hwwg-2DS (Fig. 2), was significant for AUDPC, FS, and LP in both experiments. QLr.hwwg-2DS was contributed by CI13227, located between SNP IWB34642 and IWB8545, and explained 11.2 to $20.5 \%$ of the phenotypic variation for slow-rusting resistance.

Based on the map locations of linked SSR and SNP markers, the second QTL that was associated with FS and AUDPC in the fall 2012 experiment and LP and AUDPC in the spring 2013 experiment was localized on chromosome arm 7BL and designated as $Q L r$. $h w w g-7 B L$ (Table 2). The peak of this QTL was at a 1.8-cM interval between SNP IWB9496 and IWB24039 with LOD values from 3.5 to 8.2 in the two experiments, respectively. The QTL explained 6.3 to $13.9 \%$ of the phenotypic variation for different traits, and CI13227 contributed the favorable allele.

The third QTL, designated as QLr.hwwg-7AL, was significantly associated with LP and FS in the spring 2013 experiment (Table 2). This QTL, also contributed by CI13227, was located in an interval of $10.2 \mathrm{cM}$ and flanked by SNP IWB42182 and IWB73053 (Fig. 2). QLr.hwwg-7AL explained 5.0 and $9.3 \%$ of the phenotypic variation for LP and FS, respectively.

The fourth QTL showed a minor effect on FS and AUDPC in the fall 2012 experiments (Table 2). This QTL resided in an interval of $0.4 \mathrm{cM}$ on chromosome 3B and was flanked by SNP markers IWB35536 and IWB5899 (Fig. 2). This QTL was contributed by the
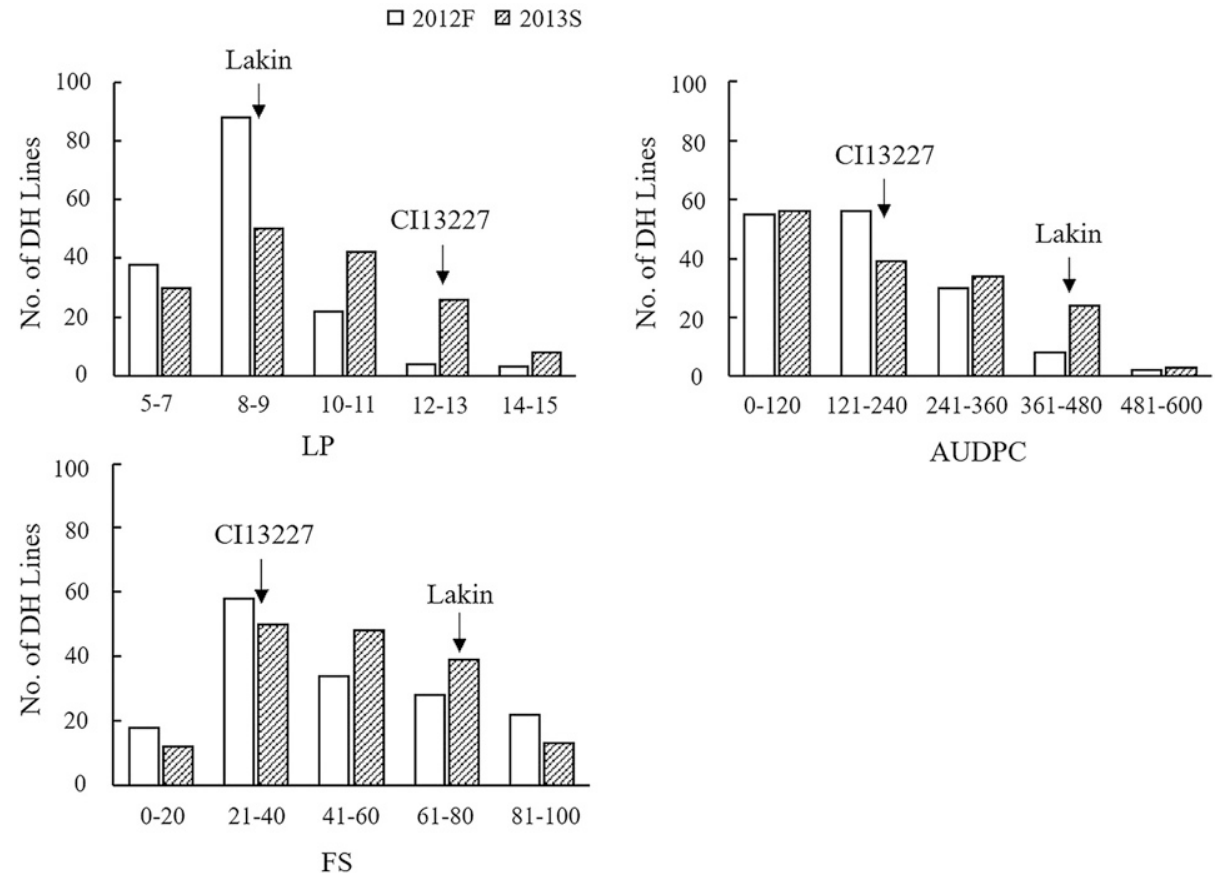

Fig. 1. Frequency distributions of final disease severity (FS), area under disease progress curve (AUDPC), and latent period (LP) for the parents CI13227 and Lakin, and the 181 doubled-haploid (DH) lines measured in the two greenhouse experiments. 
moderately susceptible parent Lakin and explained 4.2 to $5.4 \%$ of the phenotypic variation for the two traits.

Effects of QTL on leaf rust reaction. To investigate the effects of individual QTL on leaf rust reaction, DH that carried different allelic combinations at the three QTL on 2D, 7B, and 7A were grouped and their substitution effects were compared (Fig. 3). The eight possible allelic combinations of the three QTL were designated as $\mathrm{ABC}, \mathrm{ABc}, \mathrm{AbC}, \mathrm{Abc}, \mathrm{aBC}, \mathrm{aBc}, \mathrm{abC}$, and $\mathrm{abc}$, where $\mathrm{A}, \mathrm{B}$, and $\mathrm{C}$ represent marker alleles associated with the resistance alleles at the QTL on 2DS, 7BL, and 7AL of CI13227, respectively; and a, $\mathrm{b}$, and $\mathrm{c}$ represent corresponding alternate alleles from Lakin. The closest markers to the three QTL (IWB34642 on 2DS, IWB24039 on 7BL, and IWB73053 on 7A) were selected to represent the QTL. The contrasting alleles at each of the three SNP loci exhibited a 1:1 segregation ratio in the $\mathrm{DH}$ population. All eight genotypes were found in the DH population. Mean AUDPC for the eight genotypic

TABLE 2. Chromosomal locations (Chr), marker intervals (in centimorgans $[\mathrm{cM}]$ ), coefficients of determination $\left(R^{2}\right)$, additive effects (add), and logarithm of the odds (LOD) values for significant quantitative trait loci (QTL) identified in the CI13227 $\times$ Lakin doubled-haploid population

\begin{tabular}{|c|c|c|c|c|c|c|c|}
\hline Trait & $\mathrm{Chr}$ & QTL $^{\mathrm{a}}$ & Flanking markers & Interval (cM) & LOD & $R^{2}(\%)$ & Add \\
\hline LP2012F & 2D & QLr.hwwg-2DS & IWB8545-IWB34642 & 16.4 & 5.8 & 11.2 & -0.58 \\
\hline FS2012F & $2 \mathrm{D}$ & QLrhwwg-2DS & IWB8545-IWB34642 & 16.4 & 9.9 & 20.5 & 16.12 \\
\hline AUDPC2012F & 2D & QLr.hwwg-2DS & IWB8545-IWB34642 & 16.4 & 12.5 & 19.5 & 73.17 \\
\hline FS2013S & 2D & QLr.hwwg-2DS & IWB8545-IWB34642 & 16.4 & 10.4 & 17.5 & 12.92 \\
\hline AUDPC2013S & $2 \mathrm{D}$ & QLr.hwwg-2DS & IWB8545-IWB34642 & 16.4 & 12.2 & 19.3 & 81.44 \\
\hline FS2012F & 7B & QLr.hwwg-7BL & IWB9496-IWB24039 & 1.8 & 3.6 & 6.3 & 6.78 \\
\hline AUDPC2012F & 7B & QLr.hwwg-7BL & IWB9496-IWB24039 & 1.8 & 5 & 8.1 & 35.83 \\
\hline LP2013S & $7 \mathrm{~A}$ & QLr.hwwg-7AL & IWB42182-IWB73053 & 10.2 & 3.2 & 5.0 & -0.53 \\
\hline FS2013S & $7 \mathrm{~A}$ & QLr.hwwg-7AL & IWB42182-IWB73053 & 10.2 & 5.3 & 9.3 & 7.21 \\
\hline FS2012F & 3B & QLr.hwwg-3B & IWB35536-IWB5899 & 0.4 & 3.1 & 5.4 & -6.26 \\
\hline AUDPC2012F & 3B & QLr.hwwg-3B & IWB35536-IWB5899 & 0.4 & 2.7 & 4.2 & -25.95 \\
\hline
\end{tabular}

${ }^{a}$ CI13227 contributes all resistance alleles except for QLr.hwwg-3BS that was contributed by Lakin.
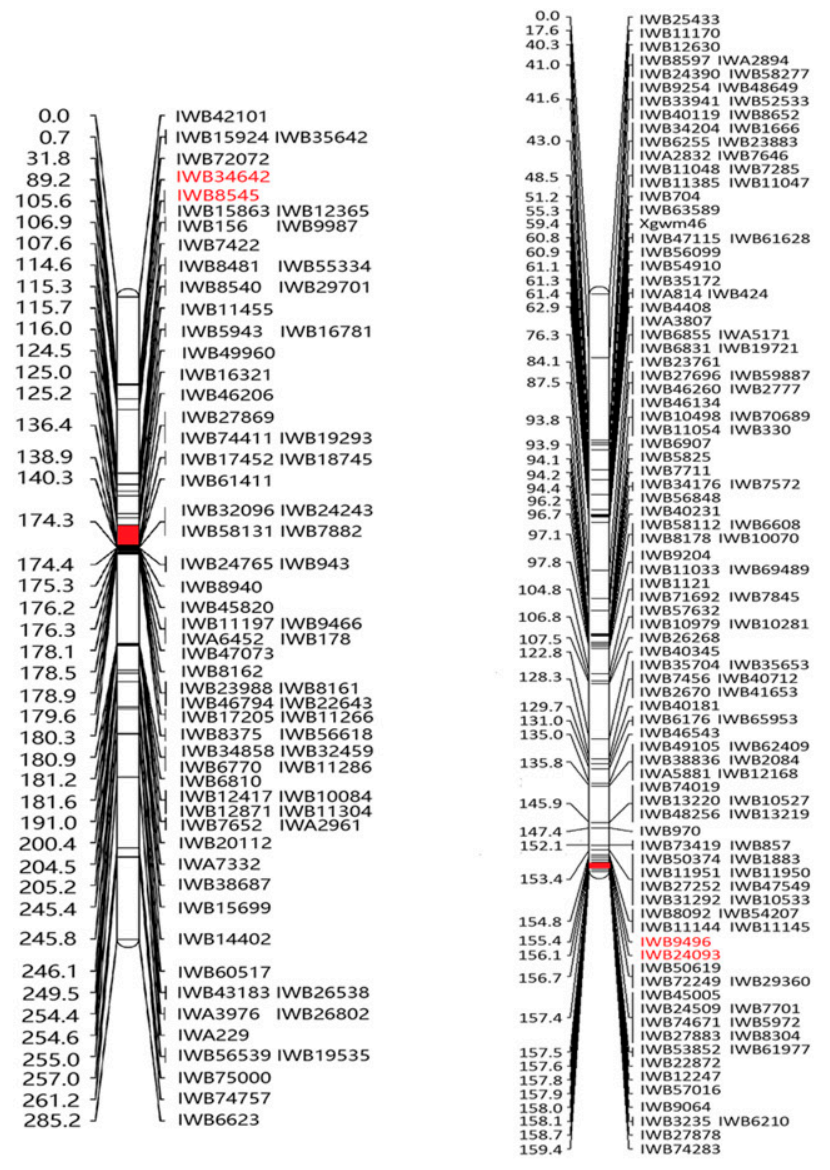

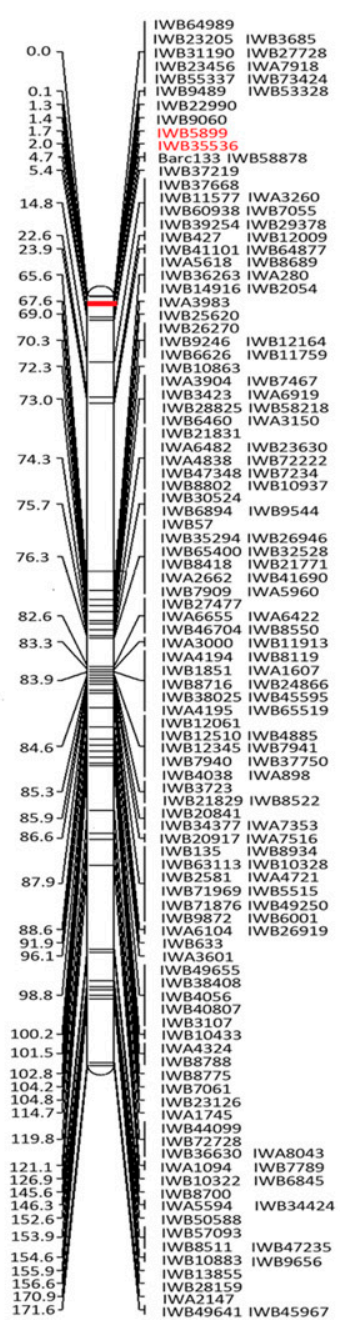

Fig. 2. High-density linkage maps of chromosomes 2D, 7B, 7A, and 3B (left to right) constructed using wheat 90K single-nucleotide polymorphism and simple sequence repeat markers. Dark bars indicate quantitative trait loci regions for slow-rusting traits detected in the doubled-haploid population of CI13227 $\times$ Lakin. 
groups of $181 \mathrm{DH}$ was 95.8 to 316.6 (Fig. 3). The mean AUDPC for the genotypic group with either $Q L r h w w g-2 D S$ (Abc) or $Q L r$. $h w w g-7 B L(\mathrm{aBc})$ was significantly lower than that of the genotypic group without any QTL (abc). The genotypic groups with $Q L r$. $h w w g-2 D S$ and an additional QTL, either QLr.hwwg-7BL or $Q L r$. $h w w g-7 A L(\mathrm{ABc}$ and $\mathrm{AbC})$, had a significantly lower mean AUDPC than genotypic groups with only one resistance QTL (Abc, aBc, and $\mathrm{abC}$ ). DH lines with all the three resistance QTL had a significantly lower mean AUDPC than that of QLr.hwwg-7AL plus an additional QTL (AbC and aBC). Thus, QLr.hwwg-2DS had the largest effect on leaf rust reaction, $Q L r . h w w g-7 B L$ the second, and $Q L r . h w w g-7 A L$ the smallest. The mean AUDPC of the groups with resistance alleles on $Q L r . h w w g-2 D S$ plus an additional QTL (ABc or $\mathrm{AbC}$ ) were consistently lower than those with $Q L r . h w w g-2 D S$ alone (Abc) or without QLr.hwwg-2DS (aBc, aBC, abC, or abc).

KASP marker conversion and verification. To verify the genotypic data generated by the $90 \mathrm{~K}$ SNP array and convert the arraybased SNP markers to breeder-friendly KASP-based SNP for MAS in breeding, 18 array-based SNP from the four QTL regions were used to design KASP assays. Among them, 11 (4 on chromosome 2DS, 2 on 7BL, 2 on 7A, and 3 on 3B) showed polymorphisms between the parents and in the DH population. Of the 11 SNP (Table 3), 8 had SNP data identical to those of the corresponding SNP from the 90K chip, and thus were remapped to the four QTL regions. Three SNP were mapped outside the QTL regions because two SNP had a different SNP call in one DH line, and one SNP had different SNP calls in five $\mathrm{DH}$ lines. The average error rate for chip-based SNP determination was $0.25 \%$.

\section{DISCUSSION}

The adult-plant slow-rusting resistance in CI13227 was mapped to three QTL: QLr.hwwg-2DS, QLr.hwwg-7AL, and QLr.hwwg$7 B L$. Resistance was quantified by measuring LP, AUDPC, and FS. The three different measures of slow-rusting resistance were highly correlated ( $r=0.89$ to 0.96 ), in agreement with previous reports

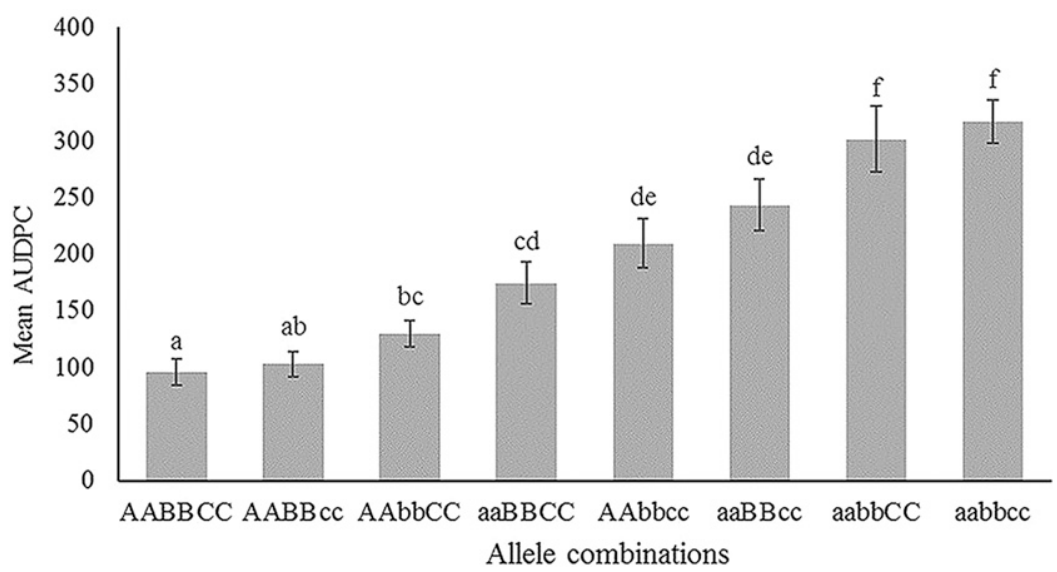

Fig. 3. Mean effects and standard errors for different combinations of quantitative trait loci (QTL) on area under disease progress curve (AUDPC). A, B, and C represent the marker alleles from CI13227 closely linked to QTL on 2DS, 7BL and 7AL, respectively; and a, b and c represent corresponding alternate alleles from Lakin, respectively. Bars with the same letter are not significantly different $(P=0.05)$ using a Duncan's multiple range test. Intervals on tops of the bars are standard errors of the means.

TABLE 3. Kompetitive allele-specific polymerase chain reaction primers based on sequences from the $90 \mathrm{~K}$ single nucleotide polymorphism arrays

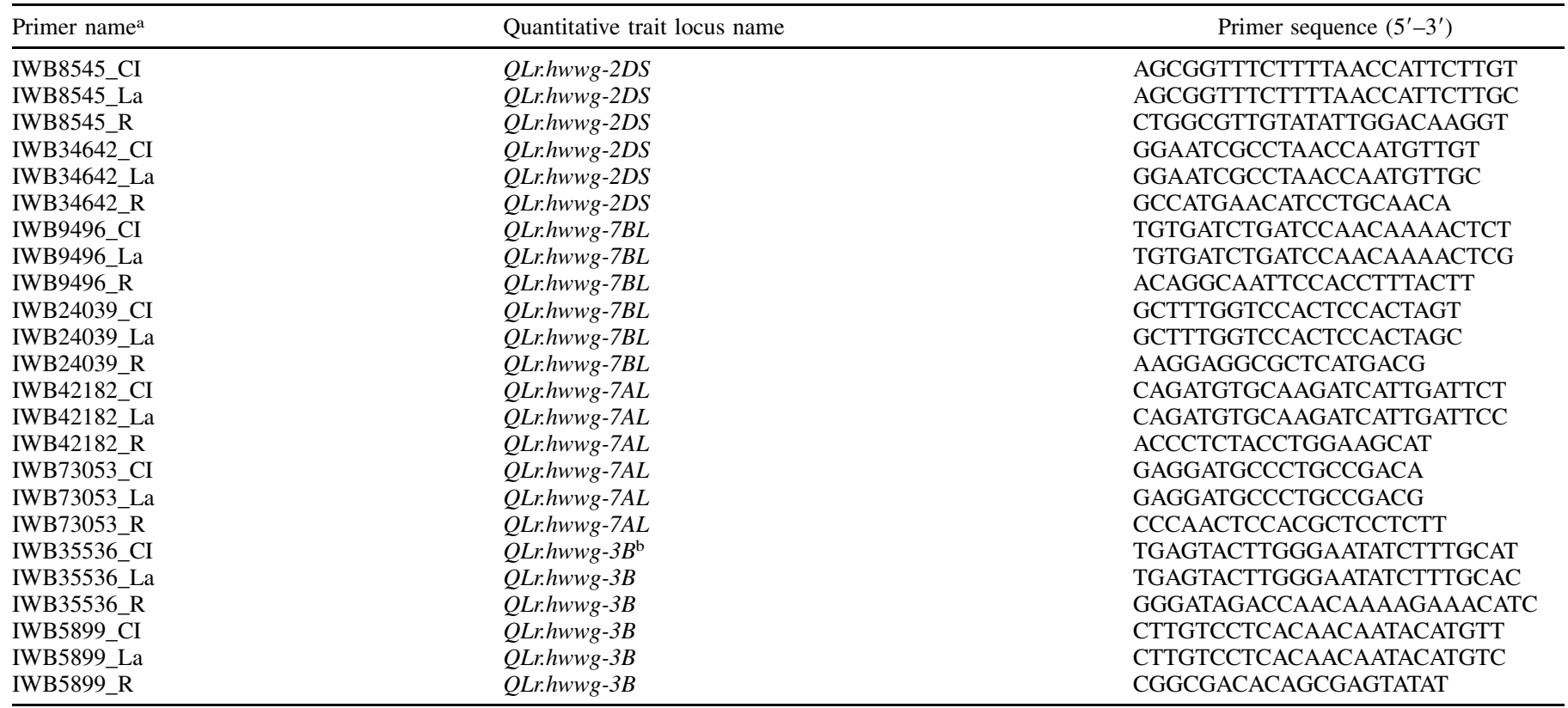

${ }^{a} \mathrm{CI}$ refers to forward primer with the CI13227 allele, La refers to forward primer with the Lakin allele, and R refers to the reverse primer.

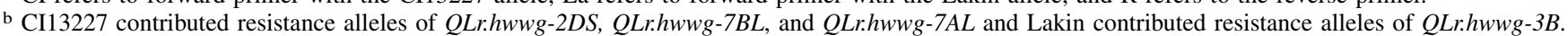


(Das et al. 1993). QTL for the different traits were mapped to the same positions, suggesting that the three traits reflected the same underlying genetic control (Table 2).

QLr.hwwg-2DS was mapped between SNP IWB8545 and IWB34642 and explained 11 to $21 \%$ of the phenotypic variation for the three traits. The location of $Q L r . h w w g-2 D S$ overlaps an LP QTL, QLrlp.osu-2DS, flanked by XTGC.CTA208 and XACTG.GTG185 (Fig. 4). QLrlp.osu-2DS is from a population derived from CI13227 $\times$ Suwon 92 reported by Xu et al. (2005c). Because the source of resistance, phenotype, and QTL location are the same, we conclude that they are identical and, thus, the slow-rusting QTL QLr.hwwg-2DS is verified in CI13227.

There are at least three other studies that have reported a similar QTL on 2DS. QLrP .sfr-2DS and QLrRI.sfr-2DS for AUDPC of disease severity and infection response, respectively, were mapped between $X c f d 53$ and $X g d m 35$ in 'Forno' (Schnurbusch et al. 2004). QLr.cim-2DS derived from 'Kundan' provided APR to leaf rust and was mapped in the region near Xbarc124 and Xcfd36 (Ren et al. 2017). Zhang et al. (2009) reported an APR QTL in CIMMYT 'Saar' between Xbarc124 and Xgwm296a. In each case, the phenotype was similar and the intervals overlapped with $Q L r . h w w g-2 D S$.

Raupp et al. (2001) mapped race-specific resistance gene $L r 39$ on 2DS at $10.7 \mathrm{cM}$ distal to marker Xgwm210. Singh et al. (2004) showed that the leaf rust resistance gene $L r 41$ was the same as $L r 39$ located on chromosome 2DS. Sun et al. (2009) mapped Lr39/41 at the end of chromosome arm 2DS at $1.0 \mathrm{cM}$ distal to Xbarc124
(Fig. 4A). In the current study, QLr.hwwg-2DS was located at least 6.0 cM proximal to Xbarc124 (Fig. 4C). Lr39/41 is highly effective in seedlings but $Q L r . h w w g-2 D S$ is an APR gene. Rust culture MFPSB is avirulent to Lr39 but it was virulent on seedlings of CI13227. Therefore, we can conclude that $L r 39 / L r 41$ and $Q L r . h w w g-2 D S$ are different genes.

Hiebert et al. (2007) reported adult-plant leaf rust resistance gene $L r 22 a$ approximately $2 \mathrm{cM}$ proximal to marker Xgwm296 on chromosome 2DS (Fig. 4D). The proximal flanking marker for $Q L r$. $h w w g-2 D S$, IWB8545, was located at $2.0 \mathrm{cM}$ distal to $\mathrm{Xgwm} 296$ in our study (Fig. 4C). Lr22a is a strong major gene and confers very high levels of resistance in the field whereas QLr.hwwg-2DS has a relatively minor slow-rusting effect that reduced FS by 13 to $16 \%$; thus, they have different phenotypes. $L r 22 b$ is an allele that is nearly universally defeated by prevalent races (McIntosh et al. 1995) and, therefore, can be excluded from consideration. Ingala et al. (2012) mapped an APR gene, designated LrSV1, in Sinvalocho MA between Xgwm261 and Xgwm296 and concluded that it could be allelic with $L r 22 a$. Although the phenotype of $L r 22 a$ is different from $Q L r . h w w g-2 D S$, and the location of QLr.hwwg-2DS appears to be distal to $L r 22 a$ (Fig. 4), there is some uncertainty about the location (Hiebert et al. 2007). Thus, we cannot yet exclude the possibility that $Q L r . h w w g-2 D S$ is allelic with APR locus Lr22a.

QLrid.osu-2DS was associated with infection duration in the CI13227 $\times$ Suwon 92 population and was flanked by XGCTG. CGCT118 proximally and Xgwm261 distally (Xu et al. 2005b). The
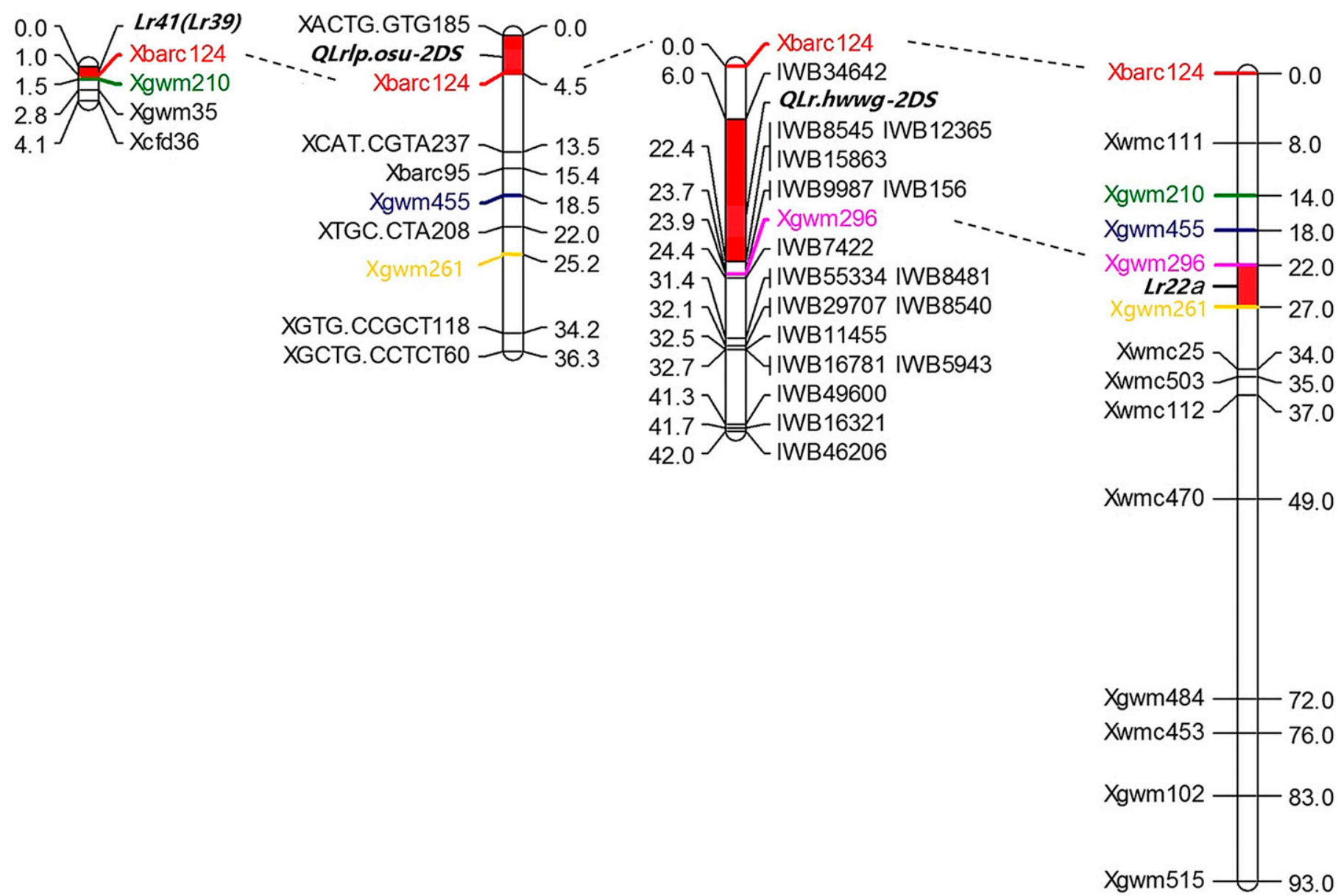

\section{A}

B

Fig. 4. Comparison of the Qlr.hwwg-2DS quantitative trait loci (QTL) map developed in the current study with three previously published genetic maps of chromosome 2DS. A, Map with Lr39/Lr41 from Sun et al. (2009); B, map with QLrlp.osu-2DS from Xu et al. (2005c); C, present QTL map of Qlr.hwwg-2DS constructed using the doubled-haploid population from the cross CI13227 × Lakin; and D, map for Lr22a from Hiebert et al. (2007). 
distal flanking marker $X g w m 261$ is at least $5 \mathrm{cM}$ from the proximal flanking marker IWB8545 of QLr.hwwg-2DS (Fig. 4). Therefore, these two QTL are not overlapping. The CI13227 allele of QLrid. $o s u-2 D S$ conferred a longer infection duration and was associated with a 7-day-later heading date than Suwon 92. Xu et al. (2005a) mapped a QTL in the same population for heading date into the same interval flanked by XGCTG.CGCT118 and Xgwm261. They postulated that the heading date QTL was associated with photoperiod sensitivity gene $P p d-D 1$. Using POPSEQ (Edae et al. 2015) and the sequences of $I W B 8545$ (Wang et al. 2014) and wheat Ppd-DI (GenBank: AB646976.1), we verified that $P p d-D 1$ is approximately $20 \mathrm{cM}$ proximal to IWB8545. Consequently, QLrid.osu-2DS is probably not related to slow-rusting resistance to leaf rust but, instead, is related to the effect of $P p d-D 1$ on heading date and epidemic development. There are many factors that can affect infection duration; therefore, it is probably not a good measure of slow-rusting resistance, especially in field studies.

Several additional leaf rust resistance loci were reported on 2DS but they differ from $Q L r . h w w g-2 D S$ in location. QLr.cimmyt-2DS from Avocet (Li et al. 2014; Rosewarne et al. 2012) was flanked by $w P T-8319$ and $w P T-3728$. Using POPSEQ and the sequence of $w P T-3728$ (http://www.diversityarrays.com/dart-map-sequences), we found that the QTL is probably on 2DL rather than 2DS. Seedling resistance locus $L r 2$, which has alleles $L r 2 a, L r 2 b$, and $L r 2 c$, was mapped proximal to $X g w m 484$ and is at least $50 \mathrm{cM}$ from QLr.hwwg$2 D S$ (Tsilo et al. 2014). In addition, $L r 15$ is closely linked or may be allelic to the $L r 2$ locus (McIntosh et al. 1995; Tsilo et al. 2014).

QLr.hwwg-7BL was mapped between SNP IWB9496 and IWB24039 near the tip of chromosome arm 7BL and explained 6 to $14 \%$ of the phenotypic variation for the three traits in the CI13227 $\times$ Lakin population. The location of QLr.hwwg-7BL overlaps an LP QTL, QLrlp. osu-7BL, flanked by XCATG.ATGC125 and XCAT.CTA155 (Fig. 5). QLrlp.osu-7BL is from the CI13227 $\times$ Suwon 92 population (Xu et al. 2005c). QLr.hwwg-7BL also overlaps QLr.osu-7BL associated with AUDPC, FS, and infection rate in the CI13227 $\times$ Suwon 92 population (Xu et al. 2005b). Because the source of resistance, phenotypes, and QTL locations are in agreement, the slow-rusting QTL, QLr.hwwg-7BL, is verified in CI13227.

Several studies have mapped a similar adult-plant slow-rusting QTL on the distal portion of 7BL (Herrera-Foessel et al. 2012; Li et al. 2014). Herrera-Foessel et al. (2012) reported Lr68 for slow rusting in 'Parula' spring wheat that was tightly linked in coupling

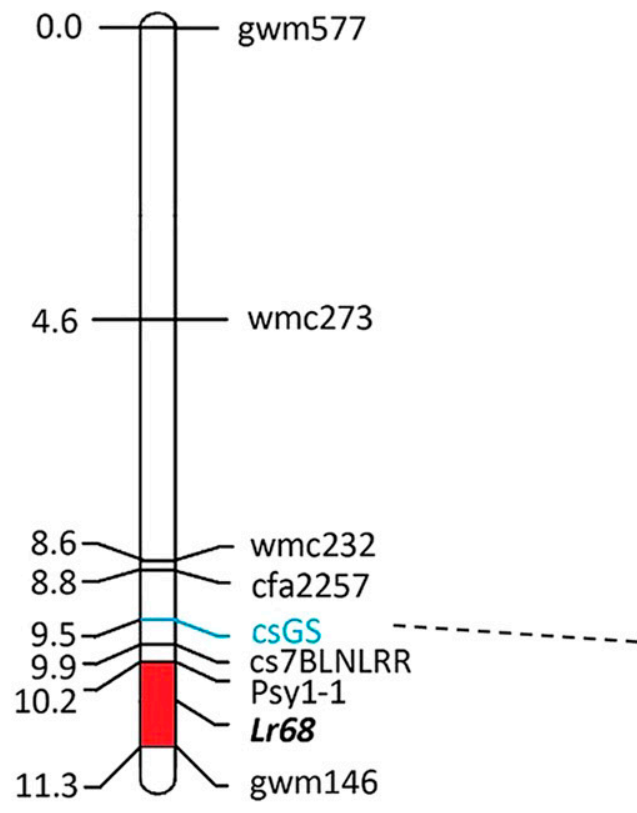

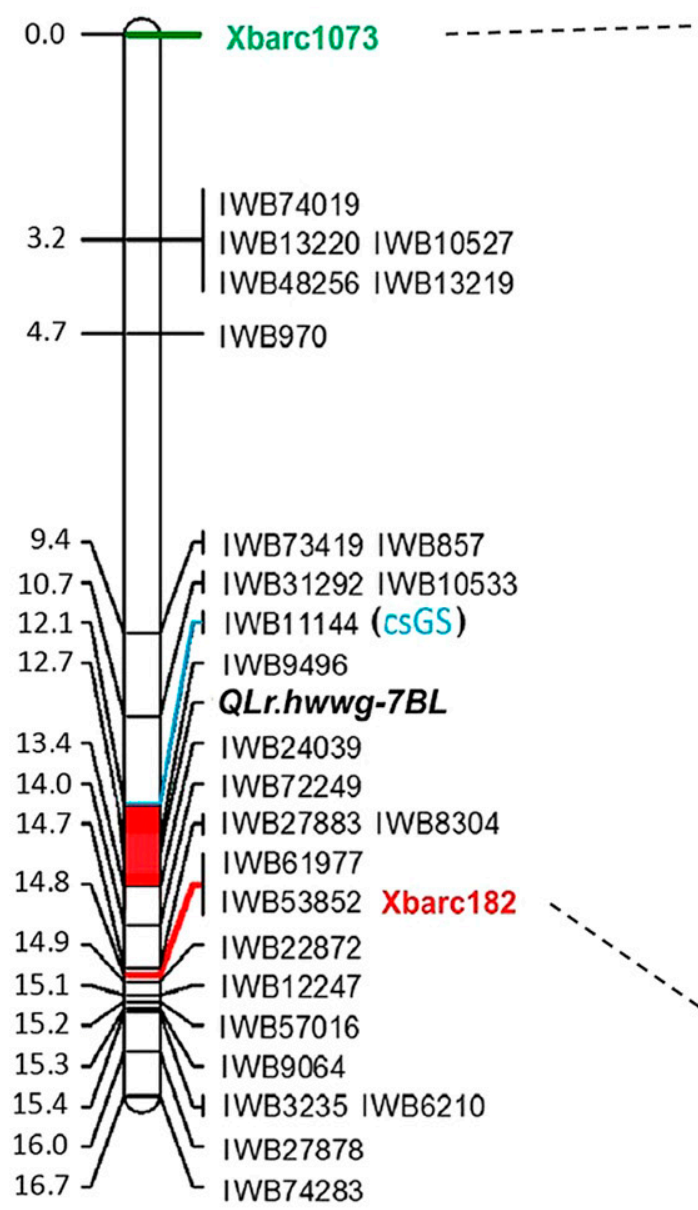

A

B

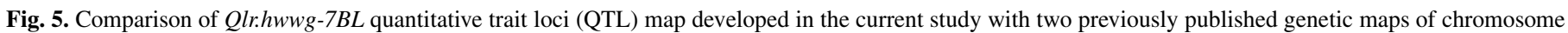

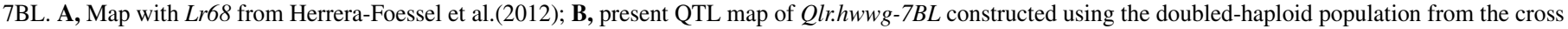
CI13227 × Lakin; and C, map with QLrlp.osu-7BL from Xu et al. (2005c). Black bars indicate QTL locations. 
to Lr14b. They tested CI13227 for seedling resistance and found it had the same "X" mesothetic IT as Lrl4b. They considered it likely that CI13227 also carries the closely linked Lr68. To confirm the relationship between $Q L r . h w w g-7 B L$ and $L r 68, c s G S$, a marker closely linked to Lr68 (Herrera-Foessel et al. 2011), was genotyped in the CI13227 $\times$ Lakin DH population in the present study. The results showed that $\operatorname{cs} G S$ explained $11 \%$ of the phenotypic variation for LP (data not shown) and was $0.3 \mathrm{cM}$ away from IWB9496, a marker flanking $Q L r . h w w g-7 B L$ (Fig. 5). Therefore, it is very likely that QLr.hwwg-7BL in CI13227 is Lr68.

The third slow-rusting QTL in CI13227 was designated QLr. hwwg-7AL and was flanked by IWB42182 and IWB73053 on chromosome arm 7AL. IWB42182 was mapped near the centromere on the Wheat_2014_90KSNP map (https://wheat.pw.usda. gov/GG3/). $Q L r . h w w g-7 A L$ was detected in only one of two experiments and was associated with an increase in LP and a reduction in FS that accounted for 5 to $9 \%$ of the phenotypic variation. We found only three reports of leaf rust resistance loci on 7AL. Lr19, which confers near immunity, is on a translocation segment from Thinopyrum ponticum in translocation line 7A/Ag\#12 (McIntosh et al. 1995). Lr20, which is defeated by nearly all North American races, is completely linked to $\mathrm{Sr} 15$ at the tip of 7AL (McIntosh et al. 1995). Tsilo et al. (2014) mapped an adult-plant leaf rust resistance gene near markers $w P t-1601$ and Xcfa2240, which are also distally located in 7AL (https://wheat.pw.usda.gov/GG3/). QLr.hwwg-7AL differs from all three previously reported loci on 7AL; thus, it is likely novel. However, it was not seen in previous studies of CI13227 (Xu et al. $2005 \mathrm{~b}, \mathrm{c})$ and needs to be verified.

A minor QTL designated QLr.hwwg-3BS was detected on chromosome 3BS and was contributed by Lakin, the moderately susceptible parent. This QTL is probably responsible for the transgressive segregation for higher resistance than CI13227 shown in Figure 1. QLr. $h w w g-3 B S$ was flanked by IWB35536 and IWB5899 and accounted for 4 to $5 \%$ of the variation in one of two experiments. The location of IWB35536 is near Lr27 and Sr2 (https://wheat.pw.usda.gov/ $\mathrm{GG} 3 /)$. However, $L r 27$ is a seedling resistance gene that requires the complementary gene Lr31 for expression (McIntosh et al. 1995). Buerstmayr et al. (2014) described a minor APR QTL, QLr.ifa-3BS, for leaf rust resistance in 'Capo' wheat on 3BS between Xbarc133 and Xgwm389. This overlaps the flanking region for QLr.hwwg$3 B S$. Ingala et al. (2012) reported an APR gene designated LrSV2 from Sinvalocho MA on 3BS completely linked to Xgwm533. This marker is within the intervals for QLr.hwwg-3BS and QLr.ifa-3BS. Therefore, it is likely that $Q L r . h w w g-3 B S$ is identical to one of the previously described APR QTL on 3BS.

Slow-rusting QTL QLrlp.osu-2B from Xu et al. (2005b,c) and QLr.osu-2B from Xu et al. (2005b) were both mapped near the centromere on chromosome $2 \mathrm{~B}$ in CI13227 and probably represent the same major APR locus. Surprisingly, a slow-rusting QTL was not detected in the current study on 2B. Xbarc 167, which is near the QTL peak for QLrlp.osu-2B and QLr.osu-2B, is mapped close to the $L r 13$ and $L r 23$ loci ((https://wheat.pw.usda.gov/GG3/). $L r 23$ is a seedling resistance gene and can be excluded from consideration. Lr13 is a race-specific resistance gene that is present in 'Klein Anniversario (McIntosh et al. 1995), one of the parents of CI13227 (Shaner and Finney 1980). Virulence to $\mathrm{Lrl} 3$ was once rare but is now common in North American isolates (Zhang et al. 2016); therefore, it is probable that our test isolate was virulent to Lr13. The simplest explanation is that the inconsistent APR QTL near the centromere on 2B in CI13227 is the race-specific APR gene Lrl3 from Klein Anniversario.

Kolmer et al. (2012) evaluated a segregating Tc*3/CI13227 backcross population in the field and mapped Lr46 in the population. However, in the current study, no QTL was identified on chromosome 1B although the diagnostic marker for Lr46, csLV46, was polymorphic in the CI13227 $\times$ Lakin population. The nonsignificant difference in all rust ratings between the RIL groups with the contrasting alleles suggests that $L r 46$ was not highly expressed under greenhouse conditions involving a monocyclic disease test. It is also possible that csLV46 is not diagnostic for Lr46 in the CI13227 $\times$ Lakin population. Kolmer et al. (2012) also mapped Lr3ka presumably derived from Klein Anniversario in CI13227. Our test isolate was virulent to $L r 3 k a$ and, therefore, was not detectable in our study. The fact that several other QTL were detected in this study but not by Kolmer et al. (2012) might be due to the different types of mapping populations used in the two studies. The Tc*3/CI13227 population used by Kolmer et al. (2012) was developed by phenotypic selection and backcrossing and, thus, these QTL were most likely lost during selection for backcrossing.

Taking account of all the evidence, the most likely $L r$ gene complement for CI13227 is Lr3ka, Lr13, Lr14b, Lr46, Lr68, QLr. $h w w g-2 D S$, and $Q L r . h w w g-7 A L$. This is an interesting combination involving several genes having slow-rusting, synergistic, and pleiotropic effects (Herrera-Foessel et al. 2012; McIntosh et al. 1995; Singh et al. 1998, 2000). All but Lr3ka and $L r l 4 b$ are APR types. Even the seedling resistance gene $L r 3 k a$ was reported to have a longer LP in seedling tests with avirulent isolates (Kolmer et al. 2012). It is impressive that this combination of the resistance genes was selected by R. M. Caldwell without the benefits of modern molecular breeding tools.

Conclusions. This study verified slow-rusting QTL QLrlp.osu$2 D S$ (here designated $Q L r . h w w g-2 D S$ ) that was found in a previous study of CI13227 × Suwon 92 (Xu et al. 2005c). QLr.osu-7BL from Xu et al. (2005c) was also verified and probably is identical to Lr68. A minor QTL from CI13227, QLr.hwwg-7AL, is probably novel but needs to be verified. A minor adult plant QTL from Lakin was designated $Q L r . h w w g-3 B S$ and is likely identical to previously reported APR QTL on 3BS that are near $S r 2$ and $L r 27$. Most of the genes in the combination have already been recognized and exploited to varying degrees in wheat breeding programs. For the future, $Q L r . h w w g-2 D S$ may be of greatest interest because it consistently accounted for up to $21 \%$ of the phenotypic variation. QLr.hwwg-7AL needs to be verified and tested in different genetic backgrounds. QLr.hwwg-3BS from Lakin is a minor QTL but is of interest because it may provide pleiotropic resistance to stripe rust (Buerstmayr et al. 2014). Near-isogenic lines can be derived to further characterize these loci using the breeder-friendly KASP markers that were developed for the QTL identified in this study (Table 3). The introgression of these QTL, especially QLr.hwwg-2DS, into commercial cultivars may significantly improve wheat leaf rust resistance and diversity of the available slow-rusting resistance QTL. The new KASP markers will facilitate marker-assisted transfer of these QTL into germplasm lines or adapted wheat cultivars.

\section{ACKNOWLEDGMENTS}

This study was funded by the National Research Initiative Competitive Grants 2011-68002-30029, 2017-67007-25939, and 2017-67007-25929 from the USDA National Institute of Food and Agriculture. Mention of trade names or commercial products in this publication is solely for the purpose of providing specific information and does not imply recommendation or endorsement by the U.S. Department of Agriculture. USDA is an equal opportunity provider and employer.

\section{LITERATURE CITED}

Bolton, M. D., Kolmer, J. A., and Garvin, D. F. 2008. Wheat leaf rust caused by Puccinia triticina. Mol. Plant Pathol. 9:563-575.

Buerstmayr, M., Matiasch, L., Mascher, F., Vida, G., Ittu, M., Robert, O., Holdgate, S., Flath, K., Neumayer, A., and Buerstmayr, H. 2014. Mapping of quantitative adult plant field resistance to leaf rust and stripe rust in two European winter wheat populations reveals co-location of three QTLs conferring resistance to both rust pathogens. Theor. Appl. Genet. 127:2011-2028.

Campbell, C. L., and Madden, L. V. 1990. Introduction to Plant Disease Epidemiology. John Wiley \& Sons, New York.

Cavanagh, C. R., Chao, S., Wang, S., Huang, B. E., Stephen, S., Kiani, S., Forrest, K., Saintenac, C., Brown-Guedira, G. L., Akhunova, A., See, D., Bai, G., Pumphrey, M., Tomar, L., Wong, D., Kong, S., Reynolds, M., da Silva, 
M. L., Bockelman, H., Talbert, L., Anderson, J. A., Dreisigacker, S., Baenziger, S., Carter, A., Korzun, V., Morrell, P. L., Dubcovsky, J., Morell, M. K., Sorrells, M. E., Hayden, M. J., and Akhunov, E. 2013. Genome-wide comparative diversity uncovers multiple targets of selection for improvement in hexaploid wheat landraces and cultivars. Proc. Natl. Acad. Sci. USA 110: 8057-8062.

Clark, J. A. 1940. Registration of improved wheat varieties, XIII. J. Am. Soc. Agron. 32:72-75.

Das, M. K., Rajaram, S., Kronstad, W. E., Mundt, C. C., and Singh, R. P. 1993. Associations and genetics of three components of slow rusting in leaf rust of wheat. Euphytica 68:99-109.

Dyck, P. L., Samborski, D. J., and Anderson, R. G. 1966. Inheritance of adultplant leaf rust resistance derived from the common wheat varieties Exchange and Frontana. Can. J. Genet. Cytol. 8:665-671.

Edae, E. A., Bowden, R. L., and Poland, J. 2015. Application of population sequencing (POPSEQ) for ordering and imputing genotyping-by-sequencing markers in hexaploid wheat. G3: Genes Genomes Genet. 5:2547-2553.

Herrera-Foessel, S. A., Lagudah, E. S., Huerta-Espino, J., Hayden, M. J., Bariana, H. S., Singh, D., and Singh, R. P. 2011. New slow-rusting leaf rust and stripe rust resistance genes $\operatorname{Lr67}$ and $\mathrm{Yr} 46$ in wheat are pleiotropic or closely linked. Theor. Appl. Genet. 122:239-249.

Herrera-Foessel, S. A., Singh, R. P., Huerta-Espino, J., Rosewarne, G. M., Periyannan, S. K., Viccars, L., Calvo-Salazar, V., Lan, C., and Lagudah, E. S. 2012. Lr68: A new gene conferring slow rusting resistance to leaf rust in wheat. Theor. Appl. Genet. 124:1475-1486.

Herrera-Foessel, S. A., Singh, R. P., Lillemo, M., Huerta-Espino, J., Bhavani, S., Singh, S., Lan, C., Calvo-Salazar, V., and Lagudah, E. S. 2014. Lr67/Yr46 confers adult plant resistance to stem rust and powdery mildew in wheat. Theor. Appl. Genet. 127:781-789.

Hiebert, C. W., Thomas, J. B., Somers, D. J., McCallum, B. D., and Fox, S. L. 2007. Microsatellite mapping of adult-plant leaf rust resistance gene $\mathrm{Lr} 22 \mathrm{a}$ in wheat. Theor. Appl. Genet. 115:877-884.

Ingala, L., López, M., Darino, M., Pergolesi, M. F., Diéguez, M. J., and Sacco, F. 2012. Genetic analysis of leaf rust resistance genes and associated markers in the durable resistant wheat cultivar Sinvalocho MA. Theor. Appl. Genet. 124: $1305-1314$

Kolmer, J. A., Lin, M., and Bai, G. 2012. Genetics of leaf rust resistance in the winter wheat line CI13227. Crop Sci. 52:2166-2172.

Kosambi, D. D. 1943. The estimation of map distances from recombination values. Ann. Eugen. 12:172-175.

Krattinger, S. G., Lagudah, E. S., Spielmeyer, W., Singh, R. P., Huerta-Espino, J., McFadden, H., Bossolini, E., Selter, L. L., and Keller, B. 2009. A putative $\mathrm{ABC}$ transporter confers durable resistance to multiple fungal pathogens in wheat. Science 323:1360-1363.

Lee, T. S., and Shaner, G. 1985. Transgressive segregation of length of latent period in crosses between slow leaf-rusting wheat cultivars. Phytopathology 75:643-647.

Li, Z., Lan, C., He, Z., Singh, R. P., Rosewarne, G. M., Chen, X., and Xia, X. 2014. Overview and application of QTL for adult plant resistance to leaf rust and powdery mildew in wheat. Crop Sci. 54:1907-1925.

Lillemo, M., Asalf, B., Singh, R. P., Huerta-Espino, J., Chen, X. M., He, Z. H., and Bjørnstad, A. 2008. The adult plant rust resistance loci Lr34/Yr18 and Lr46/Yr29 are important determinants of partial resistance to powdery mildew in bread wheat line Saar. Theor. Appl. Genet. 116:1155-1166.

McIntosh, R. A., Wellings, C. R., and Park, R. F. 1995. Wheat Rusts: An Atlas of Resistance Genes. CSIRO, Melbourne, Australia.

McIntosh, R. A., Yamazaki, Y., Dubcovsky, J., Rogers, W. J., Morris, C., Appels, R., and Xia, X. C. 2013. Catalogue of Gene Symbols for Wheat. Online publication. 12th Int. Wheat Genet. Symp. Yokohama, Japan. https:// shigen.nig.ac.jp/wheat/komugi/genes/macgene/2013/GeneCatalogueIntroduction. pdf

Moore, J. W., Herrera-Foessel, S., Lan, C., Schnippenkoetter, W., Ayliffe, M., Huerta-Espino, J., Lillemo, M., Viccars, L., Milne, R., and Periyannan, S. 2015. A recently evolved hexose transporter variant confers resistance to multiple pathogens in wheat. Nat. Genet. 47:1494-1498.

Peterson, R. F., Campbell, A. B., and Hannah, A. E. 1948. A diagrammatic scale for estimating rust intensity on leaves and stems of cereals. Can. J. Res. 26c:496-500.

Raupp, W. J., Brown-Guedira, G. L., and Gill, B. S. 2001. Cytogenetic and molecular mapping of the leaf rust resistance gene Lr39 in wheat. Theor. Appl. Genet. 102:347-352.
Ren, Y., Singh, R. P., Basnet, B. R., Lan, C. X., Huerta-Espino, J., Lagudah, E. S., and Ponce-Molina, L. J. 2017. Identification and mapping of adult plant resistance loci to leaf rust and stripe rust in common wheat cultivar Kundan. Plant Dis. 101:456-463.

Roelfs, A. P., Singh, R. P., and Saari, E. E. 1992. Rust Diseases of Wheat: Concepts and Methods of Disease Management. CIMMYT, D.F., Mexico.

Rosewarne, G. M., Singh, R. P., Huerta-Espino, J., Herrera-Foessel, S. A., Forrest, K. L., Hayden, M. J., and Rebetzke, G. J. 2012. Analysis of leaf and stripe rust severities reveals pathotype changes and multiple minor QTLs associated with resistance in an Avocet $\times$ Pastor wheat population. Theor. Appl. Genet. 124:1283-1294.

Schnurbusch, T., Paillard, S., Schori, A., Messmer, M., Schachermayr, G., Winzeler, M., and Keller, B. 2004. Dissection of quantitative and durable leaf rust resistance in Swiss winter wheat reveals a major resistance QTL in the Lr34 chromosomal region. Theor. Appl. Genet. 108: 477-484.

Shaner, G., Buechley, G., and Nyquist, W. E. 1997. Inheritance of latent period of Puccinia recondita in wheat. Crop Sci. 37:748-756.

Shaner, G., and Finney, R. E. 1980. New sources of slow leaf rusting resistance in wheat. Phytopathology 70:1183-1186.

Singh, R. P., Huerta-Espino, J., Bhavani, S., Herrera-Foessel, S. A., Singh, D., Singh, P. K., Velu, G., Mason, R. E., Jin, Y., Njau, P., and Crossa, J. 2011. Race non-specific resistance to rust diseases in CIMMYT spring wheats. Euphytica 179:175-186.

Singh, R. P., Huerta-Espino, J., Rajaram, S., Barna, B., and Kiraly, Z. 2000. Achieving near-immunity to leaf and stripe rusts in wheat by combining slow rusting resistance genes. Acta Phytopathol. Hung. 35:133-139.

Singh, R. P., Mujeeb-Kazi, A., and Huerta-Espino, J. 1998. Lr46: A gene conferring slow-rusting resistance to leaf rust in wheat. Phytopathology 88:890-894.

Singh, S., Franks, C. D., Huang, L., Brown-Guedira, G. L., Marshall, D. S., Gill, B. S., and Fritz, A. 2004. Lr41, Lr39, and a leaf rust resistance gene from Aegilops cylindrica may be allelic and are located on wheat chromosome 2DS. Theor. Appl. Genet. 108:586-591.

Sun, X., Bai, G., and Carver, B. F. 2009. Molecular markers for wheat leaf rust resistance gene Lr41. Mol. Breed. 23:311-321.

Tsilo, T. J., Kolmer, J. A., and Anderson, J. A. 2014. Molecular mapping and improvement of leaf rust resistance in wheat breeding lines. Phytopathology 104:865-870.

Van Ooijen, J. W. 2011. Multipoint maximum likelihood mapping in a full-sib family of an outbreeding species. Genet. Res. 93:343-349.

Wang, S., Wong, D., Forrest, K., Allen, A., Chao, S., Huang, B. E., Maccaferri, M., Salvi, S., Milner, S. G., Cattivelli, L., Mastrangelo, A. M., Whan, A., Stephen, S., Barker, G., Wieskeke, R., Plieske, J., International Wheat Genome Sequencing Consortium, Lilemo, M., Mather, D., Appels, R., Dolferus, R., Brown-Guedira, G., Korol, A., Akhunova, A. R., Feuillet, C., Salse, J., Morgante, M., Pozniak, C., Luo, M.-C., Dvorak, J., Morell, M., Dubcovsky, J., Ganal, M., Tuberosa, R., Lawley, C., Mikoulitch, I., Cavanagh, C., Edwards, K. J., Hayden, M., and Akhunov, E. 2014. Characterization of polyploid wheat genomic diversity using a high-density 90000 single nucleotide polymorphism array. Plant Biotechnol. J. 12:787-796.

William, M., Singh, R. P., Huerta-Espino, J., Islas, S. O., and Hoisington, D. 2003. Molecular marker mapping of leaf rust resistance gene Lr46 and its association with stripe rust resistance gene $\operatorname{Yr} 29$ in wheat. Phytopathology 93:153-159.

Xu, X., Bai, G., Carver, B. F., and Shaner, G. E. 2005a. A QTL for early heading in wheat cultivar Suwon 92. Euphytica 146:233-237.

Xu, X., Bai, G., Carver, B. F., Shaner, G. E., and Hunger, R. M. 2005b. Molecular characterization of slow leaf-rusting resistance in wheat. Crop Sci. 45:758-765

Xu, X.-Y., Bai, G.-H., Carver, B. F., Shaner, G. E., and Hunger, R. M. 2005 c. Mapping of QTLs prolonging the latent period of Puccinia triticina infection in wheat. Theor. Appl. Genet. 110:244-251.

Zhang, L., Li, Z., Lillemo, M., Xia, X., Liu, D., Yang, W., Luo, J., and Wang, H. 2009. QTL mapping for adult-plant resistance to leaf rust in CIMMYT wheat cultivar Saar. Sci. Agric. Sin. 42:388-397.

Zhang, P., Hiebert, C. W., McIntosh, R. A., McCallum, B. D., Thomas, J. B., Hoxha, S., Singh, D., and Bansal, U. 2016. The relationship of leaf rust resistance gene $\mathrm{Lr} 13$ and hybrid necrosis gene $\mathrm{Ne} 2 \mathrm{~m}$ on wheat chromosome 2BS. Theor. Appl. Genet. 129:485-93. 